\title{
Les études de Saussure sur les légendes: un rapide parcours à travers quelques interprétations
}

\author{
Pinheiro, Clemilton Lopes \\ Université Federale de Rio Grande do Norte/Post-doc CAPES/4159-13-6 \\ clemiltonpinheiro@hotmail.com
}

\section{Introduction}

Il n'y a rien de nouveau à dire que Ferdinand de Saussure s'est longuement intéressé aux légendes germaniques. Il a commencé à étudier à fond ces légendes en 1903 et continué jusqu'à $1910^{1}$. Ces recherches manuscrites s'organisent autour de deux ensembles de récits (l'épopée des Nibelungen et les aventures de Tristan) et elles ont été menées avec la minutie et le zèle qui le caractérisaient. Ces manuscrits ont fait l'objet de plusieurs éditions: Avalle (1973), Komatsu (1985), Marinetti et Merli (1986) et Turpin $(2003 \mathrm{a})^{2}$.

Plusieurs travaux donnant des interprétations sur ces études de Saussure ont été réalisés. Kim (1995) en mentionne quatre principales: Avalle (1973), Engler (1974), Prosdocimi (1983) et Arrivé (1986).

Kim fait un résumé général des idées de ces auteurs et souligne que les quatre études "présentent des thèses ou des commentaires diamétralement opposés dans leurs travaux d'interprétation” (1995: 293). Avalle (1973) propose de confronter le Saussure du Cours de Linguistique Général avec le Saussure de la légende. Pour Kim (1995: 293) "il a prétendu découvrir que dans une sémiologie saussurienne des légendes, le signe n'existe pas, et qu'au lieu du signe doué d'une structure stable, la légende n'est qu'une sorte de nuage ou nébuleuse, un agrégat d'éléments".

À l'inverse, Engler (1974) considère qu'il n’y a pas de différences essentielles entre la sémiologie linguistique et la sémiologie mythographique de la pensée saussurienne.

Prosdocine (1983) se demande si Saussure a appliqué son point de vue sémiologique à l'identification de l'être mythologique. Pour Kim (1995: 293), "la réponse de Prosdocimi est négative avérant un écart infranchissable entre le signe linguistique et le symbole légendaire. Il n'accepte ni homologie, ni analogie des faits linguistique et légendaire".

Enfin, Kim (1995: 263) cite le point de vue d' Arrivé (1986), “qui a participé à ce débat pour éclaircir la notion de symbole chez Saussure en faisant voir, en quelque sorte, dans le discours mythologique sur le symbole une autodestruction par rapport au discours linguistique".

C'est dans ce contexte d'étude que s'inscrit cet article. Nous partirons du fait que d'autres études ont été développées et nous reprendrons leur sujet afin de montrer d'autres points de vue sur les principes, les méthodes et les enjeux épistémologiques de la pratique interprétative de Saussure sur les légendes.

Afin d'atteindre ce but, nous présenterons un bref survol des quelques études qui ont été développées à partir des années 1990: Kim (1995), susmentionné, Fehr (1996), Arrivé (2001, 2012), Rastier (2009), Turpin (2003b) et Salon (2010). Nous proposerons de les faire interagir en analysant leurs positionnements et en essayant de poser d'autres questions et de nouveaux débats. Nous croyons, bien sûr, 
qu'il existe d'autres œuvres tout aussi importantes. Le choix de ces œuvres n'a pas été guidé par un critère théorique spécifique, seulement du fait de notre connaissance du sujet.

\section{Les études sur les légendes: plus lectures, autres questions}

\subsection{Relation entre sémiologie et linguistique}

D'abord, il faut remarquer que toutes les lectures mettent en place, en quelque sorte, une certain type de relation entre sémiologie et linguistique dans les études saussuriennes sur les légendes. Mais chacune le fait à différents degrés, plus proches ou plus lointains. Les réflexions de Fehr (1996) et Arrivé (2001) établissent très spécifiquement cette relation.

L'étude de Fehr (1996) concerne plus précisément la question du rapport de l'œuvre posthume de Saussure avec celle publiée de son vivant. Et dans ce cadre, il fait, bien que succinctement, un rapport entre les réflexions sémiologiques de Saussure et ses recherches sur les légendes germaniques. Selon Fehr, l'intérêt de Saussure pour le Nibelungenlied était initialement historique, "mais il se verra bientôt confronté à la problématique du signe" (1996, p. 183).

À partir de cette problématique, il semble que les réflexions sémiologiques de Saussure autour des légendes l'amènent à se convaincre d'un nouvel aspect du signe dont la sémiotique philosophique n'avait pas encore rendu compte. Suivant ce raisonnement, Fehr résume la démarche de Saussure "telle qu'elle se présente à travers ses publications, ses manuscrits et ses lettres" (p. 184) et perçoit un changement paradigmatique: "la dimension historique des faits linguistiques n'est pas éliminée, mais elle est ramenée à la question du mode d'existence normale des systèmes sémiologiques en tant que faits sociaux". Ainsi, Fehr lit les études de Saussure sur les légendes pour comprendre le projet de construction de la sémiologie, science $n^{\prime}$ existant pas encore.

A cet égard, la lecture des manuscrits et des publications qui ont précédé le CLG est particulièrement fascinante parce qu'elle permet de comprendre que la sémiologie de Saussure, en tant que science en train de se faire, n'était pas quelque chose qui devait simplement donner à la linguistique un cadre général en s'y ajoutant du dehors, mais que sous le titre de sémiologie se résumait, pour ainsi dire, tout ce qui débordait la linguistique de par son intérieur même. (Fehr, 1996: 185)

Arrivé (2001: 13), comme il le reconnaît lui-même, vise aussi, dans son article, à "réfléchir sur le problème des relations entre la linguistique et la sémiologie". Le point de départ de l'auteur pour cette réflexion est l'asymétrie entre les deux recherches.

On constate en effet que, sauf erreur ou oubli, le travail sur la légende n'est jamais allégué quand, dans le $C L G$, il est question de la sémiologie. Saussure consent parfois à donner des exemples de "systèmes de signes" autres que la langue : il les choisit alors dans les deux classes suivantes:

a) D'une part des systèmes dérivés de la langue, ou en tout cas envisagés comme tels dans l'une des deux conceptions que s'en fait Saussure. Ce sont l'écriture et l'alphabet des sourds-muets (...).

b) D'autre part des systèmes régionaux tels que les rites symboliques, les formes de politesse, les signaux militaires (...). (2001: 17) 
Dans la lecture d'Arrivé, le symbole de la légende n'a pas les caractères du signe linguistique. Le signe linguistique est un type de signe parmi d'autres et est présenté comme un objet tout à fait spécifique. Cette différence rend compte de l'absence de la légende dans la recherche sur la sémiologie linguistique.

$\mathrm{Au}$ contraire de la légende, la langue est fréquemment citée. "Ainsi, la langue est à différentes reprises explicitement donnée comme un objet de la sémiologie, en raison de sa 'parenté' avec la légende" (2001: 17). Arrivé propose une esquisse de réponse à ce problème. L'explication repose sur la conception de personnage, symbole de la légende: "Le signe ne consiste en rien. Il ne tient qu'à la rencontre provisoire et accidentelle de quelques traits voués à tout instant à se désunir. Mais pour constituer sans délai un autre signe"'(2001: 20).

Le symbole de la légende est un être inexistant, et pour cela, un paradoxe. Cependant il accède à une sorte de vie, de conscience et même de réflexion. Arrivé perçoit des "phénomènes bizarres" de la personnification du symbole dans l'écriture de Saussure qu'il interprète comme "la marque d'un désir de substance, voire de substance pensante, pour cet être inexistant" (2001: 20). Dans ce sens, il y aurait un rapport entre le signe de la langue et le symbole de la légende. Mais Arrivé assume le fait que cette réflexion de Saussure est "labyrinthique".

On découvrit en 1996 le manuscrit de Saussure, publié en 2002 sous le titre De l'essence double du langage, qui formule un programme de linguistique générale dont les notes compilées dans le CLG ne donne qu'un reflet partiel et déformé. Selon Rastier (2013), la découverte de ces manuscrits a favorisé de nouvelles interprétations de la pensée de Saussure.

Cet écrit semble pour ainsi dire le chaînon manquant qui permet de relier le Mémoire et le Cours: d'une part, il permet une compréhension unifiée des notes et fragments de linguistique générale publiés en 1974 par Engler, ou découverts conjointement à De l'essence en 1996 mais non intégrés à son dossier éditorial; mais encore il recèle des nouveautés radicales, notamment sur les dualités, comme la dualité langue/parole, et sur la dualité signifiant/signifié qui seront explorées dans les grandes recherches inédites de la décennie 1900-1910, sur les légendes germaniques et les anagrammes. (Rastier, 2003: 11-12)

Ainsi, pour Rastier (2003), les études sur les légendes et les anagrammes dépasse l'espace confiné de la grammaire. Dans un passage de De l'essence, Saussure montre que la sémiologie comprenne la morphologie, grammaire, syntaxe, synonymie, rhétorique, stylistique, lexicologie, "le tout étant inséparable" (Saussure, 2002: 45). Cette position a conséquence sur le problème des relations entre sémiologie et linguistique dans les études saussuriens.

Malgré les articles déjà publiés sur le sujet, la dépendance inversée, que Saussure a opéré entre sémiologie et linguistique, a encore besoin d'être clarifiée, ou, du moins, plus étudiée. Il s'agit alors d'une réflexion qui n'est pas inédite (on peut même se souvenir d'Engler, 1980) mais encore très importante et très étonnante dont l'origine se trouve particulièrement dans les manuscrits sur les légendes. 


\subsection{Un projet sémiologique}

Si, selon ce que nous avons déjà montré, Fehr (1996) et Arrivé (2001) mettent en comparaison sous différents aspects les projets sémiologique et linguistique de Saussure, Kim (1995) et Turpin (2003b) mettent en évidence le projet sémiologique en soulignant les points principaux qui le caractérisent.

Kim (1995) expose la conceptualité de la mythologie selon Saussure en repérant quelques instances théoriques dans son raisonnement formel. Dans sa lecture, l'auteur précise que, face à la légende, Saussure est à la fois un historien et un sémiologue. "Alors que Saussure est conscient du fait que la légende représente un mode du langage sémiologique, sa mythographie s'est portée sur les opérations que le mythe institue à partir du fondement historique" (1995: 295). Elle condense alors la position de Saussure concernant le statut sémiologique du symbole légendaire en trois points: 1) le sens du symbole, 2) ses lois de transformation et 3) son statut épistémologique.

Concernant le premier point, Kim cite Saussure afin de préciser le sens du mot symbole. "Mais dans la mythographie, Saussure considère le symbole comme une espèce de signe. Autrement dit, le symbole est aussi un signe, mais un signe particulier" (1995: 296). Les symboles de la légende subissent deux lois générales de transformation : "1) la variation modérée et limitée du symbole, c'est-à-dire qu'il ne doit ni rester fixe, ni varier indéfiniment. 2) cette altérabilité permanente du symbole s'explique par sa circulation sociale qui en modifie à chaque instant la valeur" (1995: 296).

Enfin, le critère décisif pour définir le statut épistémologique du symbole est la caractéristique sociale: "en effet, Saussure ne reconnaît comme sémiologique que la partie des phénomènes qui apparaît de manière caractéristique comme un produit social, et il refuse de considérer comme sémiologique ce qui est proprement individuel" (1995: 296).

Pour conclure sa réflexion, Kim fait la remarque suivante sur l'identité du symbole.

En effet, Saussure met en cause la valeur de l'identité dans la sémiologie mythographique, suite à l'examen des éléments constitutifs de la légende, à savoir, les actes, les fonctions, le motif, etc. Le mythographe Saussure avoue son impuissance à savoir en quoi consistera l'identité, à quel signe on doit la reconnaitre et la proclamer (1995: 297).

Turpin (2003b) appelle les études de Saussure sur les légendes projet sémiologique. Pour elle, "le Saussure linguiste est donc un Saussure ethnographe et historien de la langue, sans qu'il faille voir là deux Saussure" (2003b: 308). Dans son article, Turpin esquisse le modèle sémiologique de Saussure, non développé, qu'elle appelle sémiologie du discursif.

Dans ce modèle, le symbole légendaire n'est pas une unité fixe, qui a une identité stable. "Pour Saussure une unité ne saurait symboliser une autre unité parce qu'il y a processus sémiologique et que toute unité est prise dans un réseau d'association 'd'un côté ou d'un autre' - forme ou sens” (2003b: 312).

La légende pour Saussure est un processus de symbolisation qui rend compte d'une série d'opérations d'associations et de transformations. Ces opérations sont faites à partir du mythe, de la légende ou de l'histoire dans un jeu de symboles entre identités et différences, qui "peut être réglé, mais peut aussi échapper à la rationalité des oppositions" (2003b: 312). L'analyse du processus de symbolisation consisterait alors en la recherche des invariants des légendes et leurs règles de transformations. 
Le modèle peut être résumé comme suit.

Une unité, une identité, est donc constituée d'un plexus de différences qui s'organisent en un discours (légendes, mythes, histoire mise en récit), ce discours lui-même étant plexus d'autres discours, tant il est vrai que tout discours renvoie à d'autres discours par diverses chaînes associatives: une légende peut renvoyer à une autre légende par une sorte de contaminations de traits, mais aussi à d'autres types de discours qui ont pu la précéder chronique ou mythes se croisent alors en elle par ce travail de mise en discours qu'a pu faire chaque fois un récitant, en introduisant les mêmes variations souvent involontaires que l'on retrouve dans le travail de cet autre discours social qu'est la rumeur. (Turpin, 2003b: 314)

Toutefois, Turpin perçoit des points communs entre ce modèle et celui développé par Saussure dans les cours de linguistique générale. Pour elle, comme pour Kim d'ailleurs (1995), la légende chez Saussure est un autre mode du langage sémiologique. "En fait, c'est comme une trame discursive par laquelle Saussure rattache la légende au mythe: le mythe est la trame de la légende (un peu comme l'analogie peut être la trame des mots de la langue)" (Turpin, 2003b: 312). Saussure est parti d'un problème pour chercher un modèle explicatif. Il semble que ce modèle est ce que Turpin appelle "sémiologie du discursif".

Comme nous l'avons d'abord dit, il semble que dans la lecture de Kim et Turpin la question la plus importante ne soit pas la relation entre sémiologie et linguistique, bien qu'elle soit présente, mais les principes et les enjeux épistémologiques d'une sémiologie saussurienne. Si on accepte alors de qualifier ce projet de Saussure de sémiologique, c'est ici qu'intervient une réflexion sur la relation entre ce projet et et l'émergence de la sémiotique discursive française. Nous rappelons qu'Arrivé (2007) esquisse déjà l'héritage saussurien, tel qu'il a été repris et développé par Barthes et Greimas.

\subsection{Une relation entre langue et texte}

Les études de Rastier (2009) et Salmon (2010) exposent la relation entre la langue et le texte. Selon les autres auteurs, les composantes de la réflexion saussurienne sont constituées par la recherche sur la langue et sur la légende ou mythologie. Il n'est pas utilisé le mot texte pour se référer aux légendes. Dans son interprétation, Salmon (2010: 181) se réfère à 'l'approche de l'intertextualité qui avait été initiée par Saussure". Rastier (2009: 14) se réfère aux "études textuelles". D'ailleurs, le titre de l' article est "Saussure et les textes - de la philologie des textes saussuriens à la théorie saussurienne des textes" (c'est nous que souligons).

L'étude de Rastier (2009: 1) "prend sa source dans deux séries de questions: la première porte sur la théorie des textes chez Saussure et secondairement dans le saussurisme; la seconde sur la lecture des textes saussuriens". En ce qui concerne la première série de questions, l'auteur réexamine les études textuelles conduites par Saussure, c'est à dire les anagrammes et les légendes germaniques. En fonction de l'objectif de notre article nous ne commenterons que l'examen des études sur les légendes.

D'un côté, Rastier reprend l'analyse de Turpin (2003). Il esquisse aussi d'une manière générale les principaux points du "modèle" interprétatif de Saussure des légendes. "L'entité linguistique n'est plus une simple occurrence d'un type, elle varie sans limite selon les places et les instants" (2009: 18). 
D'un autre côté, Rastier présente, en fait il ajoute, une seconde position: "ces études témoignent d'une véritable pensée de la textualité" (2009: 18). L'auteur ne définit pas de manière claire ce qu'on entend par "textualité". Mais il dit que les études "recherchent les normes de composition de légendes, y compris dans leurs transformations généralisées, sans négliger le problème de leurs racines historiques, par le biais de leur rapport avec la linguistique externe" (2009: 21). La textualité correspond à une relation sémiotique fondamentale et elle est un axe d'études textuelles "qui relie le mot, le passage, le texte et le corpus" (2009: 21).

Par cette lecture, il semble que Rastier propose que l'on doit à Saussure d'avoir unifié théorie du signe et théorie du texte dans une même discipline.

La linguistique saussurienne reste révolutionnaire parce que c'est une linguistique du texte et non une linguistique du signe: non qu'elle ait cherché à faire une 'grammaire de texte', mais parce que la définition même des unités grammaticales est renvoyée à la dimension du texte où ces unités, autrement vides et indéfinissables, prennent leur valeur. (2009: 21)

Le travail de Salmon (2010) aborde spécifiquement le rapport entre la légende et l'histoire. Dans cette approche, comme il a déjà été dit, l'auteur développe les notions de texte et d'intertextualité.

En montrant que la formation des légendes obéit à des opérations complexes de remaniement de textes antérieurs par déplacement de motifs, transfert d'attributs entre personnages ou fusion de sources hétérogènes, Saussure jette les fondements d'une approche novatrice de l'intertextualité (2010: 169).

Cette lecture de la pratique interprétative de Saussure sur les légendes montre un "nouveau mode de comparaison du texte" qui s'appelle "l'intertextualité constituante". Pour l'auteur, ce mode de comparaison des textes propose d'identifier sous un texte un autre texte qui lui sert de patron. Les textes présentent des correspondances systématiques qui peuvent s'étendre aux détails en apparence les plus insignifiants.

La comparaison des textes donne priorité aux considérations formelles sur les considérations historiques. Par ailleurs, des textes très éloignés d'un point de vue historique, et même culturel, sont confrontés afin d'établir un bilan complet des coïncidences et des divergences. Suivant ce mode de comparaison, pour Salmon, Saussure a découvert une liste de correspondances entre le mythe de Thésée et la légende de Tristan et il a proposé une hypothèse de genèse originale: "l'histoire de Tristan aurait été composée par un clerc britannique qui aurait cherché à adapter le récit de Thésée sur un modèle national" (2010: 171).

Salmon met en évidence également la réflexion de Saussure sur le degré de signification et de correspondances suffisantes autant que sur les autres difficultés méthodologiques du mode de comparaison des textes.

A ces difficultés déjà considérables s'ajoute un obstacle supplémentaire. De fait, les correspondances que Saussure identifie entre les Nibelungen et l'histoire burgonde ou entre Tristan et Thésée ne sont jamais parfaites: elles sont toujours accompagnées d'altérations qui peuvent être selon les cas plus ou moins importantes. La majeure partie des passages théoriques retrouvés dans les cahiers de Saussure est consacrée à la question suivante : quels sont les critères qui permettent d'affirmer qu'on retrouve le même épisode ou le même personnage entre les textes qu'on compare? (Salmon, 2010: 173) 
Salmon n'explique pas le changement terminologique entre légende et texte. Mais il ne fait aucun doute que l'objet de sa réflexion est le texte, bien que dans un sens non précisé. Nous croyons alors que l'interprétation de Rastier et Salmon ne pose pas une question seulement terminologique mais aussi conceptuelle. Dans ce cas il y a beaucoup de questions à penser. La première et principale question est la conception du texte qui est mise en place dans la recherche sur la légende.

Du fait de la méconnaissance, en France, de la linguistique du texte de Coseriu (Coseriu, 1994, 2007), nous nous permettons de rappeler ses positions qui permettent d'aider à la réflexion (Nous traduisons les citations qui suivent). "En ce qui concerne les catégories de la linguistique du texte, le consensus est encore plus faible parce que chaque auteur présente généralement des termes nouveaux (...). Jamais le concept de texte n'est le même pour différents auteurs, ni même parfois pour le même auteur "' (Coseriu, 2007: 83-4).

Coseriu distingue alors trois conceptions du texte, et par conséquent, trois types de linguistique du texte:

$\mathrm{Au}$ départ, il y a deux sortes de linguistiques du texte. Les objets de la première sont les textes comme niveau autonome du langage, indépendamment de la langue qui les exprime. (...). Le deuxième type de linguistique du texte a comme objet le texte en tant que niveau de structuration idiomatique. Pour cette raison, et aussi pour le bénéfice de la clarté terminologique, elle sera appelée grammaire du texte ou grammaire transphrastique (ou encore analyse transphrastique). (...) Hormis ces deux types, considérés pleinement légitimes, il existe une troisième orientation qui peut également être considérée comme linguistique du texte. (...) Mais pour celle ci, le concept de texte n'est pas clairement présenté: on fait référence au texte général, c'est à dire au texte comme possibilité universelle du langage ${ }^{4}$. (Coseriu, 2007: 116-17)

Ainsi, si l'on conçoit qu'il y a une quelconque approche linguistique du texte dans les études de Saussure sur les légendes, il importe de préciser ces distinctions. Puis il faudrait chercher le type ou plus précisément les types de relations possibles entre langue et texte.

Suivant la lecture de Rastier (2009), on peut enquêter sur deux principes de la linguistique saussurienne à partir de ses études textuelles: le primat du global (le texte) sur le local (la forme) et le primat méthodologique de la parole sur la langue. Concernant le premier primat, selon l'auteur, "Saussure énonce ce principe en raison du caractère fondamentalement contextuel et donc textuel de sa sémiotique" (2009: 14). Concernant le deuxième primat, il souligne qu'“un texte est donc une manifestation de la parole et la linguistique des textes relève de la linguistique de la parole" (2009: 14).

Selon Bronckart (2011: 350-51), la mise en œuvre effective de l'activité langagière et de la langue universelle par les groupes humains, dans des circonstances historiques et géographiques variables, requiert la prise en compte de trois entités, qui constituent les véritables objets d'une science du langage proposée par Saussure.

(a) Les textes/discours, comme premier lieu de vie des signes, dans le cadre desquels les valeurs de ces signes se re-fabriquent en permanence.

(b) La langue interne, comme système d'organisation psychologique des valeurs signifiantes extraites des textes, système qui est soumis aux contraintes conventionnelles de la langue normée dont attestent les textes, mais qui est marque aussi par l'histoire de vie et les propriétés particulières 
des personnes; cette langue interne constituant un deuxième lieu de vie des signes.

(c) La langue normée, comme système d'organisation des valeurs signifiantes des signes géré cette fois par les groupes sociaux et soumis à leurs normes propres de fonctionnement. Il s'agit là d'un troisième lieu de vie des signes, à caractère second ou abstrait: la "langue française", par exemple, n'existe qu'en tant que produit d'un travail de généralisation et d'abstraction, travail aux résultats variables et incertains comme chacun le sait.

En ces termes, Brockart semble proposer ainsi une relation entre langue et texte dans la pensée saussurienne. Il reste à savoir et à bien préciser en quelle mesure ces relations sont présentes dans les études sur les légendes.

Il reste à propos de la recherche de Saussure sur les légendes à mener une réflexion sur le statut auquel le texte accède dans sa pensée (si on le trouve): un objet linguistique ou sémiologique (dans le sens général de sémiologie), comme le fait, par exemple, Badir (1998) à propos de la notion de texte chez Hjelmslev.

\subsection{Une relation entre légende et littérature}

Dans son article Arrivé (2012) se demande comment Saussure conçoit la littérature. L'auteur examine le sort qui est réservé à la littérature dans les différents travaux de Saussure: sa recherche proprement linguistique (Cours de linguistique générale et Écrits de linguistique générale, par exemple), la recherche sémiologique sur la légende germanique et la recherche sur les anagrammes. "Nous allons donc essayer de naviguer ensemble, à propos de la littérature, dans l'océan tourmenté de la réflexion de Saussure" (2012: 34). En fonction du notre but, nous commenterons seulement la réponse prenant en compte l'examen des études sur les légendes.

Arrivé (2012: 45) pose bien la question: "le texte légendaire est-il considéré par Saussure comme littéraire?". Nous rappelons encore une fois qu'il faut bien préciser la conception du texte. Mais Nous laisserons la question dans l'ombre pour analyser comment Arrivé lit la réponse de Saussure. L'auteur interprète certains passages du texte de Saussure (ce qui est normal) et conclut que la réponse "est variable, évolutive et de ce fait ambiguë” (2012: 45).

Dans le passage du cas de Nibelungenlied,, il semble que Saussure neutralise l'opposition entre légende et littérature: "il est à la fois légendaire et littéraire" (2012: 46). Dans un autre passage, quand Saussure se réfère à la lecture de Don Quichotte, il dit que le texte littéraire est intangible, et se distingue de ce fait à la fois de la langue, de l'écriture et de la légende. Le texte littéraire se distingue de la légende, ainsi que de la langue et de l'écriture - modes de langage sémiologique -, parce qu'il est fixé par le nom de son auteur, c'est à dire "il n'est pas soumise aux avatars conjoints du temps et de la socialisation qui sont indispensables à la nature même de l'objet sémiologique" (2012: 47).

Arrivé souligne que cette affirmation de Saussure aurait été un obstacle à la construction de la sémiologie littéraire (obstacle auquel Barthes n'a jamais fait allusion). Cependant, pour Arrivé, ce passage est capital parce qu'à la fois il a un aspect négatif (la littérature échappe à la sémiologie) mais aussi des aspects positifs "qu'on peut, après Saussure, donner pour nous aider à penser la littérature" (2012: 47).

Il est intéressant de transcrire la citation exacte car elle ouvre une possibilité d'interprétation de la réflexion saussuriene sur les légendes.

1. Première retouche possible. Il n'est sans doute pas impossible d'envisager une analyse synchronique de l'objet littéraire, en dépit de son caractère non 
évolutif. Il faudrait poser un objet sémiologique spécifique non soumis aux altérations du temps, mais propre à être décrit selon les procédures de l'analyse sémiologique. Paradoxal, certes, dans l'appareil conceptuel saussurien. Mais après tout, la description synchronique d'une langue ne se donne-t-elle pas comme règle d'en ignorer les états antécédents?

2. Seconde retouche possible. Toujours en restant à l'intérieur du système saussurien, il n'est sans doute pas impossible de se donner les différentes lectures du même texte comme si elles constituaient les évolutions propres à le faire considérer comme une sémiologie. On entrerait alors dans une sémiologie, si on tient à conserver le terme saussurien, non plus du texte en lui-même, mais des lectures qui en sont à chaque instant faites. (2012: 48)

La place de la littérature chez Saussure a été intérêt de l'ouvrage de Bédouret-Larraburu \& Gisèle (2012). Dans um compte rendu consacré à cette ouvrage, Garet-Gaudek (2013) montre que les travaux se donnent pour perspective l'utilité ou l'efficacité des concepts saussuriens pour réfléchir à la littérarité. Ils concentrent leur réflexions sur trois points: la littérature chez Saussure comme objet de travail, les implications de la théorie du langage de Saussure pour la théorie de la littérature et une mise à l'épreuve de son œuvre théorique dans l'étude des textes littéraires eux-mêmes.

Garet-Gaudek (2013) souligne la divergence qui se révèle entre les points de vue lorsqu'on examine ce que fait chacun de la notion du littéraire chez Saussure. La différence, "pour ne pas dire l'opposition" entre les points de vue, "tient à la définition de la littérature adoptée par chacun, et au concept saussurien à partir duquel le littéraire va être abordé” (2013: 10).

Il est finalement intéressant de souligner la remarque suivante de Garet-Gaudek: "La confrontation des articles pointe donc vers cette conclusion, toujours provisoire: seule la perspective du discours semble aujourd'hui permettre de dire que Saussure nous aide à penser la littérature" (2013: 28). Il s'agit alors d'une réflexion encore très importante et très étonnante dont l'origine se trouve aussi dans les manuscrits sur les légendes.

\section{Mots de conclusion}

Dans cet article, nous avons parcouru, bien que très partiellement, les travaux et les interprétations sur les études que Saussure a développées sur les légendes germaniques. Nous avons réalisé la lecture de ces travaux et nous les avons organisés à partir du degré de proximité de positions des auteurs sur le sujet. Les auteurs suivent différents trajets de lecture (comme on pouvait s'y attendre). Un ensemble de réflexions établit très précisément la relation entre sémiologie et linguistique d'un point de vue théorique. Un autre ensemble essaie d'établir les principes d'un modèle sémiologique. Il y a certains auteurs qui inaugurent un regard textuel, et enfin ceux qui proposent une lecture littéraire.

Certaines de leurs interprétations reprennent des questions déjà débattues mais en apportent aussi des nouvelles: quelles sont, en fait, les relations entre les fondements du Saussure linguiste et du Saussure légendiste? Quelle distinction faire entre linguistique et sémiotique? Y a-t-il une linguistique unifiant les signes et les textes? Le texte est-il objet de la linguistique ou de la sémiologie? La sémiologie qui est pris pour objet est-elle une sémiologie littéraire? Quelle est la relation entre légende et littérature? Comment 
penser la relation entre linguistique et littérature?. Sans doute ces questions montrent l'importance et la pertinence de nouveaux débats autour de cet ensemble de manuscrits.

Selon Fenoglio (2013: 41), "l'observation des manuscrits qui préparent, réfléchissent, tel un miroir, l'écriture d'un discours particulier, permet non seulement de mettre au jour un processus de genèse scripturale mais aussi un processus de naissance conceptuelle, d'invention textuelle". Dans le cas de Saussure, cette tâche constitue particulièrement un grand défi parce qu'il n'utilise pas de concepts bien définis, mais des concepts qui attendent une formulation et des fondements.

Fenoglio souligne ainsi que la démarche de recherche de la genèse circonscrit et analyse ensuite l'avanttexte. "Avant-texte désigne, comme son nom l'indique, tout ce qui se passe avant la production du texte écrit et donc des traces attestant d'une mise en acte cognitive et graphique directement liée au texte final produit" (2013: 42). Dans le cas de Saussure, il n'y a pas de "texte final produit", mais il y a beaucoup de concepts et de fondements à formuler.

C'est pour cette raison que nous concluons par la citation d'Arrivé (2001, p. 23): "La pensée de Saussure reste - et, par la force des choses restera définitivement - en gestation et en transformation".

\section{Références bibliographiques}

Arrivé, M. (1986). Linguistique et psychanlyse, Paris, Méridiens.

Arrivé, M. (2001). La sémiologie saussurienne entre le CLC et la recherche sur la légende. Linx, 44, 13-27.

Arrivé, M. (2007). À la recherche de Ferdinand de Saussure. Paris: PUF.

Arrivé, M. (2012). De la lettre à la littérature: un trajet saussurien. In: Bedouret, S. et Prignitz, G. (éds). Linguiste et littérature 1. Saussure. En quoi Saussure peuti-il nous aider à Penser la littérature?. Pau : Presses de 1'Université de Pau et des Pays de 1 Ádour, 33-50.

Avalle, D. (1973). La sémiologie de la narrativité chez Saussure, in: Bouazis, C. (éd.), Essai de la théorie du text

Paris, Galilée, 17-49.

Badir, S. (1998). La notion de texte chez Hjelmslev. Texto! [en ligne]. Disponible sur : <http://www.revuetexto.net/Dialogues/Debat_Hjelmslev/Badir_Notion.html> (Consultée le 13/11/2013).

Bédouret-Larraburu, S. \& Prignitz, G. (éds). (2012). En quoi Saussure peut-il nous aider à penser la littérature? Pau:

Presses de l'Université de Pau et des Pays de l'Adour.

Bronckart, J. P. (2011). Une science du langage pour une science de l'humain. DELTA, 27:2, 337-61.

Coseiru, E. (1994). Textlinguistik: Eine Einführung. Tübingen: Francke.

Coseriu, E. (2007). Lingüistica del texto. Introducción a la hermenéutica del sentido (édition et annotation d'Oscar Loureda Lamas). Madrid: Arco/Libros.

Engler, R. (1974). Sémiologies saussuriennes. De l'existence du signe, Cahier Ferdinand Saussure, 29, 45-73.

Engler, R. (1980). Sémiologies saussuriennes, 2. Le canevas. Cahiers Ferdinand de Saussure, 34, 3-16.

Fehr, J. (1996). Saussure: cours, publications, manuscrits, lettres et documents. Les contours de l'oeuvre posthume et ses rapports avec l'oeuvre publiée. Histoire Épistémologie Langage. Tome 18, fascicule 2, 179-199. 
Fenoglio, I. Éléments pour une genèse de la notion d'énonciation chez Benveniste. Ce que dévoilent les manuscrits. In:

Dufaye, L. Et Gournay, L. (éds). Benveniste après demi-siècle. Regards sur l'énonciation aujourd'hui. Paris: Ophrys, 2013, 41-83

Garet-Gaudek, E. (2013). Saussure aujourd'hui, de l'écrit au discours, Acta fabula, vol. 14, n 7 . Disponible sur: http://www.fabula.org/revue/document8137.php, (consultée le 10 mars 2014).

Kim, S. (1995). La mythologie saussurienne: une ouverture sémiologique, Linx, 7, 293-300.

Komatsu, E. (1985). Tristan - Notes de Saussure. Essays and Studies, XXXII, 149-229.

Marinetti, A. y Meli, M. (1986). Ferdinand de Saussure : la leggende germaniche. Este : Zielo.

Prosdocimi, A. (1983). Sul Saussure delle leggende germaniche, Cahier Ferdinand Saussure, 37, 35-106.

Rastier, F. (2009). Saussure et les textes - de la philologie des textes saussuriens à la théorie saussurienne des textes. Texto!, XIV, n. 3, 01-27.

Rastier, F. (2003). De l'essence double du langage, um projet révélateur. Arena Romanistica, 12, 6-28.

Salmon, G. (2010). Les conditions d'une science de l'intertextualité: réflexion sur les apories du comparatisme saussurien. Rivista Italiana di Filosofia del Linguaggio, 3, 169-182.

Saussure, F. (2002). Écrits de linguistique générale. Édition par Bouquet, S. et Engler, R. Paris: Gallimard.

Turpin, B. (2003a). Légendes et récits d'Europe du Nord: de Sigfrid à Tristan. in : Bouquet, S. (éd.) Saussure. Paris: L'Herne, 351-429.

Turpin, B. (2003b). Légendes - Mythes - Histoire. La circulation des signes. in : Bouquet, S. (éd.) Saussure. Paris: L'Herne, 307-316.

\footnotetext{
${ }^{1}$ Selon Turpin, quelques dates fugurent dans les manuscrits saussuriens. "Ces quelques dates nous montrent que ces recherches étaient commencées em 1903 et occupaient Saussure em 1910” (2003a: 352).
} 
${ }^{2}$ Ces manuscrits sont conservés dans le Département des manuscrits de la Biblioteque de Genève et sont repartis en 3 lots: 8 cahiers, 383 feuilles (cote Ms. Fr. 3958/1 à 8), 10 cahiers, 228 feuilles (cote Ms. Fr. 3959/1 à 10) et 228 feuilles volantes (cote Ms. Fr. 3959/11). Selon Turpin (2003a: 353), les notes comprennent deux types de matériaux: "les matériaux servant à étayer h'hypothèse: releve factuel de coïncidences, de corresponances; les matériaux théoriques et ceux faisant allusion à um ouvrage (titres, plans, préface....)".

3 "Respecto de las categorías de la lingüística del texto, el acuerdo es, si cabe, aún menor, pues cada autor suele añadir términos nuevos (...). Tampoco el concepto de texto es idéntico en los diversos autores, y a veces ni siquiera lo es para un mismo autor".

4 "Inicialmente pueden distinguirse dos tipos de lingüística del texto. El objeto de la primera son los textos como un nivel del lenguaje autónomo, independiente de la lengua en la que se expresen. (...).La segunda clase de lingüística del texto tiene su objeto en el texto en tanto que nivel de estructuración idiomática. Por eso, y también en beneficio de la claridad terminológica, se denominará gramática del texto o gramática transoracional (también análisis transoracional o transfrástico). (...).Junto a estas dos clases de lingüística del texto que deben considerarse plenamente legítimas existe una tercera orientación que también pretende ser una lingüística del texto. (...).Lo que no se explica con claridad es de cuál de los dos conceptos de texto presentados se parte, aunque a lo hace referencia normalmente es al texto en general, esto es, al texto como posibilidad universal del lenguaje". 